\title{
Molecular characterization and new genotypes of Enterocytozoon bieneusi in minks (Neovison vison) in China
}

\author{
Wei Cong ${ }^{1}$, Si-Yuan Qin ${ }^{2}$, and Qing-Feng Meng ${ }^{3,4, *}$ \\ 1 Marine College, Shandong University at Weihai, Weihai, Shandong Province 264209, PR China \\ 2 General Station for Surveillance of Wildlife Diseases \& Wildlife Borne Diseases, State Forestry Administration (SFA), \\ Shenyang 110034, PR China \\ 3 Jilin Inspection and Quarantine Technology Center, Changchun, Jilin Province 130062, PR China \\ 4 College of Animal Science and Technology, Jilin Agricultural University, Changchun, Jilin Province 130118, PR China
}

Received 12 April 2018, Accepted 24 June 2018, Published online 20 July 2018

\begin{abstract}
Microsporidiosis is an emerging and opportunistic disease, and Enterocytozoon bieneusi is the main cause of this disease in humans. Little information is available on prevalence and genotyping of $E$. bieneusi in minks. We collected 559 feces samples of minks from Heilongjiang and Jilin provinces in 2017, and studied E. bieneusi prevalence by nested PCR. A total of 23 out of 559 minks (4.1\%) were detected as E. bieneusi-positive, and were raised in five of the seven investigated farms. Age was the only risk factor associated with $E$. bieneusi prevalence in investigated minks through logistic regression analysis. Sequence analysis of the ITS gene revealed that five E. bieneusi ITS genotypes, including Peru11, EbpC, and three novel genotypes (HLJM-1, HLJM-2 and JLM-1) were present, suggesting minks may be a potential source of human microsporidiosis.
\end{abstract}

Key words: Enterocytozoon bieneusi, Epidemiology, Genotyping, Minks, China.

\begin{abstract}
Résumé - Caractérisation moléculaire et nouveaux génotypes d'Enterocytozoon bieneusi chez les visons (Neovison vison) en Chine. La microsporidiose est une maladie émergente et opportuniste, et Enterocytozoon bieneusi est la principale cause de la microsporidiose humaine. Peu d'informations sur la prévalence et le génotypage d'E. bieneusi chez les visons sont disponibles. 559 échantillons d'excréments de visons provenant des provinces de Heilongjiang et de Jilin ont été collectés en 2017 et la prévalence d'E. bieneusi a été étudiée par PCR nichée. Au total, $23(4,1 \%)$ des 559 visons ont été détectés positifs pour E. bieneusi, et provenaient de cinq des sept fermes étudiées. L'âge était le seul facteur de risque, étudiés par analyse de régression logistique, associé à la prévalence d'E. bieneusi chez les visons. L'analyse de la séquence du gène ITS a révélé que cinq génotypes ITS d'E. bieneusi, y compris Peru11, EbpC, et trois nouveaux génotypes (HLJM-1, HLJM-2 et JLM-1), étaient présents, ce qui suggère que les visons pourraient être une source potentielle de microsporidiose humaine.
\end{abstract}

\section{Introduction}

Minks are a species of high economic importance: the animals are widely raised for their fur in Northern China, including in Heilongjiang and Jilin provinces. Minks can serve as reservoirs for many pathogens including influenza viruses [7], Aleutian mink disease virus [17], thrombocytopenia syndrome virus [21], Pentatrichomonas hominis [14], and Toxoplasma gondii [30]. Because minks are in close contact with their feeders, they can transmit many pathogens to humans, including Toxoplasma gondii [30]. Despite this, data regarding

\footnotetext{
*Corresponding author: messicw@163.com
}

the prevalence and genotypes of Enterocytozoon bieneusi in minks are scarce.

The Microsporidia contains over 1300 named species, and has a worldwide distribution. Enterocytozoon bieneusi is the most frequent causative agent of human microsporidiosis $[1,11]$ and is responsible for more than $90 \%$ of human infections [3]. E. bieneusi can infect a variety of invertebrates and vertebrates [27, 28, 33], and can be transmitted through the anthroponotic, zoonotic, water-borne, and/or food-borne routes [4, 13, 19]. The symptoms of microsporidiosis caused by E. bieneusi are diarrhea and abdominal pain in immunodeficient individuals, while the infection appears asymptomatic 
Table 1. Prevalence of Enterocytozoon bieneusi in minks in Jilin and Heilongjiang provinces, China.

\begin{tabular}{llccccc}
\hline Factor & \multicolumn{1}{c}{ Category } & No. tested & No. positive & Prevalence $(\%)(95 \% \mathrm{CI})$ & $p$-value & OR $(95 \% \mathrm{CI})$ \\
\hline Region & Heilongiiang Province & 257 & 10 & $3.9 \%(1.5-6.3)$ & 0.81 & Reference \\
& Jilin Province & 302 & 13 & $4.3 \%(2.0-6.6)$ & $1.1(0.5-2.6)$ \\
Gender & Female & 279 & 9 & $3.2 \%(1.2-5.3)$ & 0.29 & $0.6(0.3-1.5)$ \\
& Male & 280 & 14 & $5.0 \%(2.4-7.6)$ & Reference \\
Age & $\leq 3$ months & 244 & 5 & $2.0 \%(0.3-3.8)$ & 0.03 & Reference \\
& $>3$ months & 315 & 18 & $5.7 \%(3.2-8.3)$ & $2.9(1.1-7.9)$ \\
Total & & 559 & 23 & $4.1 \%(2.5-15.8)$ & \\
\hline
\end{tabular}

in immunocompetent individuals who can shed spores into the environment and become a potential source of infection for other individuals [29].

More than 240 E. bieneusi genotypes have been defined based on the internal transcribed spacer (ITS) region of the rRNA gene [1]. All the genotypes can be grouped into 9 groups (named groups 1-9). The majority of human infections are caused by the zoonotic group $1[6,15]$. However, some genotypes (such as I, J and BEB4) from the other zoonotic groups have also been found in humans [8].

In order to determine whether minks can be infected by E. bieneusi, and to assess the zoonotic risk of E. bieneusi between minks and humans, a total of 559 mink feces samples were collected from seven farms in Heilongjiang and Jilin provinces. The samples were tested to detect the prevalence of E. bieneusi and associated genotypes in minks by nested PCR amplification of the ITS region of E. bieneusi.

\section{Materials and methods}

\section{Ethics statement}

All animals were handled in strict accordance with good animal practices according to the Animal Ethics Procedures and Guidelines of the People's Republic of China, and the study was approved by the Ethics Committee of Jilin Agricultural University.

\section{Specimen collection}

In all, 559 farmed mink fecal samples were randomly collected from Heilongjiang $\left(43^{\circ} 26^{\prime} \sim 53^{\circ} 33^{\prime} \mathrm{N}, 121^{\circ} 11^{\prime} \sim\right.$ $\left.135^{\circ} 05^{\prime} \mathrm{E}\right)$ and Jilin $\left(41^{\circ} \sim 46^{\circ} \mathrm{N}, 122^{\circ} \sim 131^{\circ} \mathrm{E}\right)$ provinces, northeastern China in 2017. More than 200 minks were bred at each farm, and the sampling percentage ranged from $5 \%$ to $10 \%$ on the different farms. Fresh dejections were immediately collected using a polyethylene glove, and were then stored in ice boxes and transported to the laboratory. The Farm ID, gender and age of minks were obtained from the owners.

\section{DNA extraction and PCR amplification}

The commercial E.Z.N.A. ${ }^{\circledR}$ Stool DNA Kit (Omega Biotek Inc., Norcross, GA, USA) was used to extract genomic DNA, following the manufacturer's instructions, and extracted DNA was stored at $-20{ }^{\circ} \mathrm{C}$. PCR targeting the ITS region was used to explore the prevalence and genotypes of E. bieneusi. All the PCR operations have been described in a previous study [31]. In each trial, positive and negative controls were present. The amplification products were observed using UV light after electrophoresis in a $1.5 \%$ agarose gel containing GoldView ${ }^{\mathrm{TM}}$ (Solarbio, Beijing, China).

\section{Sequence and phylogenetic analyses}

Sangon Biotech Company (Shanghai, China) was contracted to sequence the PCR products. Sequence accuracy was evaluated by bidirectional sequencing. Replicates were made when new sequences were found (single nucleotide substitutions, insertions or deletions). ClustalX 1.83 was used to align the sequences. The neighbor-joining (NJ) method (Kimura 2-parameter model, 1000 replicates) was used to reconstruct the phylogenetic trees with Mega 5.0 software. Representative nucleotide sequences were deposited in GenBank under accession numbers MH052578-MH052582.

\section{Statistical analysis}

Data analysis of the prevalence of $E$. bieneusi infection in minks by age, gender, and different farms groups was performed by $\chi^{2}$ testing using SAS version 9.1 (SAS Institute, Cary, NC, USA) [16, 32]. When $p<0.05$, the results were considered statistically significant. Odds ratios (ORs) and their $95 \%$ confidence intervals $(95 \% \mathrm{CIs})$ were estimated to explore the strength of the association between E. bieneusi-positivity and the conditions investigated.

\section{Results and discussion}

In the present study, 23 out of 559 minks (4.1\%) were tested as E. bieneusi-positive. Female minks had a lower E. bieneusi infection rate than males (Table 1). The prevalence of E. bieneusi was 3.9\% in minks from Heilongjiang Province, and $4.3 \%$ in minks from Jilin Province (Table 1). Minks aged more than three months had a significantly higher infection rate than those aged less than three months (Table 1). The E. bieneusi prevalence in the different investigated farms ranged from $0 \%$ to $7.5 \%$ (Table 2). Sequence analysis of the ITS region revealed that five E. bieneusi ITS genotypes (two known genotypes Peru11 and EbpC; three novel genotypes HLJM-1, HLJM-2 and JLM-1) were present in investigated minks (Fig. 1). 


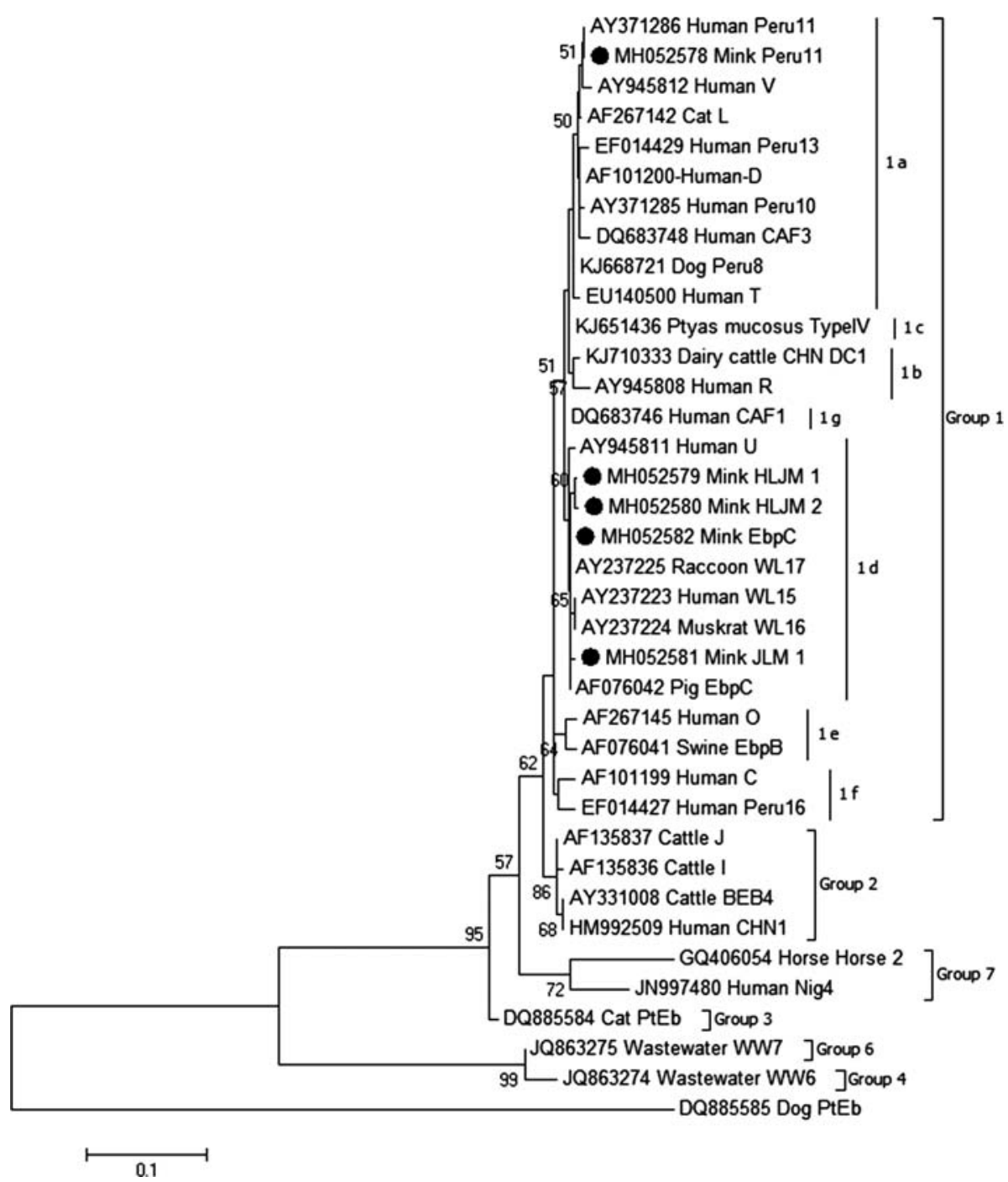

Fig. 1. Phylogenetic analyses of Enterocytozoon bieneusi using the neighbor-joining (NJ) method (Kimura 2-parameter model). Bootstrap values below $50 \%$ from 1000 replicates are not shown. E. bieneusi isolates identified in the present study are pointed out by solid circles.

Table 2. Distribution of Enterocytozoon bieneusi genotypes on different farms.

\begin{tabular}{lccl}
\hline Farm ID & Sample size & Prevalence (\%) & \multicolumn{1}{c}{ Genotype (No.) } \\
\hline 1 & 124 & 3.2 & HLJM-1 (1); Peru11 (3) \\
2 & 133 & 4.5 & HLJM-1 (1); HLJM-2 (1); Peru11 (4) \\
3 & 93 & 7.5 & Peru11 (4); EbpC (3) \\
4 & 32 & 0 & - \\
5 & 75 & 6.7 & Peru11 (2); EbpC (3) \\
6 & 78 & 0 & - \\
7 & 24 & 4.2 & JLM-1 (1) \\
Total & 559 & 4.1 & Peru11 (13); EbpC (6); HLJM-1 (2); HLJM-2 (1); JLM-1 (1) \\
\hline
\end{tabular}

The overall infection rate of E. bieneusi in minks was $4.11 \%$, which was higher than that in domestic rabbits $(0.9 \%, 4 / 426)$ [32] in Jilin, pet chinchillas $(3.6 \%, 5 / 140)$ [18] in Beijing, Zhengzhou, Anyang and Guiyang, and similar to the $4.1 \%$ infection rates in raccoon dogs (2/49) [29] in Liaoning, Heilongjiang and Jilin, and $4.6 \%$ prevalence in captive snakes $(11 / 240)$ [10] in Guangxi. However, this rate was significantly lower than that in captive golden snub-nosed monkeys $(46.2 \%, 74 / 160)[26]$ in Beijing, Shanghai, Anhui and Shanxi, and captive Asiatic black bears (27.4\%, 29/106) [24] in Sichuan and Guizhou. The difference in E. bieneusi prevalence may be related to feeding conditions, sampling time, sample sizes, and animal husbandry practices, as well as variable susceptibility of different animals. Enterocytozoon 
bieneusi is a commonly enteric pathogen, and also exists extensively in the environment. Enterocytozoon bieneusi accumulation could occur throughout a minks lifetime. Therefore, minks aged more than 3 months $(\mathrm{OR}=2.9,95 \%$ CI $1.1-7.9, d f=1$, $p=0.03$ ) were at a 2.9 times higher risk of E. bieneusi infection compared to minks less than three months of age.

More than $50 \mathrm{E}$. bieneusi ITS genotypes have been found in captive animals in China [2, 5, 9, 10, 12, 18, 22-26, 29, 31, 32]. However, only five E. bieneusi ITS genes (two known genotypes, Peru11 and EbpC; and three novel genotypes, HLJM-1, HLJM-2 and JLM-1) were found in the present research (Table 2). Genotype Peru11 (distributed on four farms) was the most frequently found genotype of all four genotypes, and was responsible for $56.5 \%$ of all infections; genotype $\operatorname{EbpC}(n=6,26.1 \%)$ and HLJM-1 $(n=2,8.7 \%)$ were found on two farms; HLJM-2 $(n=1,4.3 \%)$ and JLM-1 $(n=1,4.3 \%)$ were only identified on farm 2 and farm 7 , respectively (Table 2). These findings suggest that the five genotypes were prevalent in investigated minks in Heilongjiang and Jilin, China. The Peru11 genotype was also found in captive non-human primates, laboratory macaques, and $\mathrm{EbpC}$ was prevalent in nonhuman primates and blue foxes in China [5, 9, 12, 18, 22-26, 29, 31, 32], which suggests that E. bieneusi may be transmitted among these captive animals.

The ITS sequence analysis demonstrated that the sequence of Accession Nos. MH052578 and MH052582 was identical to that of genotypes Peru11 (Accession No. KT922238) and EbpC (Accession No. KX905207), respectively. Moreover, all the five identified E. bieneusi genotypes were grouped into group 1 (Fig. 1). Peru11 was sub-classified into group 1a (Fig. 1); EbpC, HLJM-1, HLJM-2 and JLM-1 were sub-classified into group 1d (Fig. 1). More importantly, Peru11 and EbpC were also found in HIV-positive patients in Henan [20]. These findings suggest that minks may play an important role in human infections. Although no evidence of human microsporidiosis outbreaks originating from minks or other animals has been found, we should pay close attention to nosocomial transmission among humans, minks and other animals.

\section{Competing interests}

The authors declare that they have no competing interests.

Acknowledgements. Project support was provided in part by the Natural Science Foundation of Shandong Province (Grant No. ZR2017PC004), and the State Key Laboratory of Veterinary Etiological Biology, Lanzhou Veterinary Research Institute, Chinese Academy of Agricultural Sciences (Grant No. SKLVEB2017KFKT007).

\section{References}

1. Deng L, Li W, Zhong Z, Chai Y, Yang L, Zheng H, Wang W, Fu H, He M, Huang X, Zuo Z, Wang Y, Cao S, Liu H, Ma X, Wu K, Peng G. 2018. Molecular characterization and new genotypes of Enterocytozoon bieneusi in pet chipmunks (Eutamias asiaticus) in Sichuan province, China. BMC Microbiology, 18, 37.

2. Deng L, Li W, Zhong Z, Gong C, Cao X, Song Y, Wang W, Huang X, Liu X, Hu Y, Fu H, He M, Wang Y, Zhang Y, Wu K, Peng G. 2017. Multi-locus genotypes of Enterocytozoon bieneusi in captive Asiatic black bears in southwestern China: High genetic diversity, broad host range, and zoonotic potential. PLoS One, 12, e0171772.

3. Desportes I, Le Charpentier Y, Galian A, Bernard F, CochandPriollet B, Lavergne A, Ravisse P, Modigliani R. 1985. Occurrence of a new microsporidan: Enterocytozoon bieneusi n.g., n. sp., in the enterocytes of a human patient with AIDS. Journal of Protozoology, 32, 250-254.

4. Didier ES. 2005. Microsporidiosis: an emerging and opportunistic infection in humans and animals. Acta Tropica, 94(1), 61-76.

5. Du SZ, Zhao GH, Shao JF, Fang YQ, Tian GR, Zhang LX, Wang RJ, Wang HY, Qi M, Yu SK. 2015. Cryptosporidium spp., Giardia intestinalis, and Enterocytozoon bieneusi in captive non-human primates in Qinling Mountains. Korean Journal of Parasitology, 53, 395-402.

6. Hu Y, Feng Y, Huang C, Xiao L. 2014. Occurrence, source, and human infection potential of Cryptosporidium and Enterocytozoon bieneusi in drinking source water in Shanghai, China, during a pig carcass disposal incident. Environmental Science \& Technology, 48(24), 14219-14227.

7. Jiang W, Wang S, Zhang C, Li J, Hou G, Peng C, Chen J, Shan H. 2017. Characterization of H5N1 highly pathogenic mink influenza viruses in eastern China. Veterinary Microbiology, 201, 225-230.

8. Jiang Y, Tao W, Wan Q, Li Q, Yang Y, Lin Y, Zhang S, Li W. 2015. Erratum for Jiang et al., 2015. Zoonotic and potentially host-adapted Enterocytozoon bieneusi genotypes in sheep and cattle in Northeast China and an increasing concern about the zoonotic importance of previously considered ruminant-adapted genotypes. Applied and Environmental Microbiology, 81, 5278.

9. Karim MR, Wang R, Dong H, Zhang L, Li J, Zhang S, Rume FI, Qi M, Jian F, Sun M, Yang G, Zou F, Ning C, Xiao L. 2014. Genetic polymorphism and zoonotic potential of Enterocytozoon bieneusi from nonhuman primates in China. Applied and Environmental Microbiology, 80, 1893-1898.

10. Karim MR, Yu F, Li J, Li J, Zhang L, Wang R, Rume FI, Jian F, Zhang S, Ning C. 2014. First molecular characterization of enteric protozoa and the human pathogenic microsporidian, Enterocytozoon bieneusi, in captive snakes in China. Parasitology Research, 113, 3041-3048.

11. Li J, Luo N, Wang C, Qi M, Cao J, Cui Z, Huang J, Wang R, Zhang L. 2016. Occurrence, molecular characterization and predominant genotypes of Enterocytozoon bieneusi in dairy cattle in Henan and Ningxia, China. Parasites \& Vectors, 9, 142.

12. Li W, Deng L, Yu X, Zhong Z, Wang Q, Liu X, Niu L, Xie N, Deng J, Lei S, Wang L, Gong C, Zhou Z, Hu Y, Fu H, Xu H, Geng Y, Peng G. 2016. Multilocus genotypes and broad hostrange of Enterocytozoon bieneusi in captive wildlife at zoological gardens in China. Parasites \& Vectors, 9, 395.

13. Li W, Song Y, Zhong Z, Huang X, Wang C, Li C, Yang H, Liu H, Ren Z, Lan J, Wu K, Peng G. 2017. Population genetics of Enterocytozoon bieneusi in captive giant pandas of China. Parasites \& Vectors, 10(1), 499.

14. Li X, Li J, Zhang X, Yang Z, Yang J, Gong P. 2017. Prevalence of Pentatrichomonas hominis infections in six farmed wildlife species in Jilin, China. Veterinary Parasitology, 244, 160-163.

15. Ma J, Li P, Zhao X, Xu H, Wu W, Wang Y, Guo Y, Wang L, Feng Y, Xiao L. 2015. Occurrence and molecular characterization of Cryptosporidium spp. and Enterocytozoon bieneusi in dairy cattle, beef cattle and water buffaloes in China. Veterinary Parasitology, 207, 220-227. 
16. Meng QF, Yao GZ, Qin SY, Wu J, Zhang XC, Bai YD, Cong W, Wang WL. 2017. Seroprevalence of and risk factors for Neospora caninum infection in yaks (Bos grunniens) in China. Veterinary Parasitology, 242, 22-23.

17. Prieto A, Fernández-Antonio R, Díaz-Cao JM, López G, Díaz P, Alonso JM, Morrondo P, Fernández G. 2017. Distribution of Aleutian mink disease virus contamination in the environment of infected mink farms. Veterinary Microbiology, 204, 59-63.

18. Qi M, Luo N, Wang H, Yu F, Wang R, Huang J, Zhang L. 2015. Zoonotic Cryptosporidium spp. and Enterocytozoon bieneusi in pet chinchillas (Chinchilla lanigera) in China. Parasitology International, 64, 339-341.

19. Thellier M, Breton J. 2008. Enterocytozoon bieneusi in human and animals, focus on laboratory identification and molecular epidemiology. Parasite, 15, 349-358.

20. Wang L, Xiao L, Duan L, Ye J, Guo Y, Guo M, Liu L, Feng Y. 2013. Concurrent infections of Giardia duodenalis, Enterocytozoon bieneusi, and Clostridium difficile in children during a cryptosporidiosis outbreak in a pediatric hospital in China. PLoS Neglected Tropical Diseases, 7, e2437.

21. Wang GS, Wang JB, Tian FL, Zhang HJ, Yin FF, Xu C, Xu D, Huang YT, Yu XJ. 2017. Severe fever with Thrombocytopenia Syndrome Virus infection in Minks in China. Vector Borne and Zoonotic Diseases, 17(8), 596-598.

22. Xu C, Ma X, Zhang H, Zhang XX, Zhao JP, Ba HX, Rui Du, Xing XM, Wang QK, Zhao Q. 2016. Prevalence, risk factors and molecular characterization of Enterocytozoon bieneusi in raccoon dogs (Nyctereutes procyonoides) in five provinces of Northern China. Acta Tropica, 161, 68-72.

23. Yang H, Lin Y, Li Y, Song M, Lu Y, Li W. 2017. Molecular characterization of Enterocytozoon bieneusi isolates in laboratory macaques in north China: zoonotic concerns. Parasitology Research, 116(10), 2877-2882.

24. Yang Y, Lin Y, Li Q, Zhang S, Tao W, Wan Q, Jiang Y, Li W. 2015. Widespread presence of human-pathogenic Enterocytozoon bieneusi genotype D in farmed foxes (Vulpes vulpes) and raccoon dogs (Nyctereutes procyonoides) in China: first identification and zoonotic concern. Parasitology Research, 114, 4341-4348.
25. Yang Z, Zhao W, Shen Y, Zhang W, Shi Y, Ren G, Yang D, Ling H, Yang F, Liu A, Cao J. 2016. Subtyping of Cryptosporidium cuniculus and genotyping of Enterocytozoon bieneusi in rabbits in two farms in Heilongjiang Province, China. Parasite, 23, 52.

26. Yu F, Wu Y, Li T, Cao J, Wang J, Hu S, Zhu H, Zhang S, Wang R, Ning C, Zhang L. 2017. High prevalence of Enterocytozoon bieneusi zoonotic genotype $\mathrm{D}$ in captive golden snub-nosed monkey (Rhinopithecus roxellanae) in zoos in China. BMC Veterinary Research, 13, 158.

27. Yue DM, Ma JG, Li FC, Hou JL, Zheng WB, Zhao Q, Zhang XX, Zhu XQ. 2017. Occurrence of Enterocytozoon bieneusi in donkeys (Equus asinus) in China: a public health concern. Frontiers in Microbiology, 8, 565.

28. Zhang XX, Cong W, Lou ZL, Ma JG, Zheng WB, Yao QX, Zhao Q, Zhu XQ. 2016. Prevalence, risk factors and multilocus genotyping of Enterocytozoon bieneusi in farmed foxes (Vulpes lagopus), Northern China. Parasites \& Vectors, 9, 72.

29. Zhao W, Zhang W, Yang Z, Liu A, Zhang L, Yang F, Wang R, Ling H. 2015. Genotyping of Enterocytozoon bieneusi in farmed blue foxes (Alopex lagopus) and raccoon dogs ( $\mathrm{Nyc}$ tereutes procyonoides) in China. PLoS One, 10, e0142611.

30. Zheng WB, Zhang XX, Ma JG, Li FC, Zhao Q, Huang SY, Zhu XQ. 2016. Molecular detection and genetic characterization of Toxoplasma gondii in farmed minks (Neovison vison) in Northern China by PCR-RFLP. PLoS One, 11(11), e0165308.

31. Zhang XX, Cong W, Lou ZL, Ma JG, Zheng WB, Yao QX, Zhao Q, Zhu XQ. 2016. Prevalence, risk factors and multilocus genotyping of Enterocytozoon bieneusi in farmed foxes (Vulpes lagopus), Northern China. Parasites \& Vectors, 9, 72.

32. Zhang XX, Jiang J, Cai YN, Wang CF, Xu P, Yang GL, Zhao Q. 2016. Molecular characterization of Enterocytozoon bieneusi in domestic rabbits (Oryctolagus cuniculus) in Northeastern China. Korean Journal of Parasitology, 54, 81-85.

33. Zhang XX, Shi W, Zhang NZ, Shi K, Li JM, Xu P, Zhao Q, Du R. 2017. Prevalence and genetic characterization of Toxoplasma gondii in donkeys in northeastern China. Infection, Genetics and Evolution, 54, 455-457.

Cite this article as: Cong W, Qin S \& Meng Q: Molecular characterization and new genotypes of Enterocytozoon bieneusi in minks (Neovison vison) in China. Parasite, 2018, 25, 34.

Reviews, articles and short notes may be submitted. Fields include, but are not limited to: general, medical and veterinary parasitology; morphology, including ultrastructure; parasite systematics, including entomology, acarology, helminthology and protistology, and molecular analyses; molecular biology and biochemistry; immunology of parasitic diseases; host-parasite relationships; ecology and life history of parasites; epidemiology; therapeutics; new diagnostic tools.

All papers in Parasite are published in English. Manuscripts should have a broad interest and must not have been published or submitted elsewhere. No limit is imposed on the length of manuscripts.

Parasite (open-access) continues Parasite (print and online editions, 1994-2012) and Annales de Parasitologie Humaine et Comparée (1923-1993) and is the official journal of the Société Française de Parasitologie. 